\title{
ESR2 Status by Immunohistochemistry
}

National Cancer Institute

\section{Source}

National Cancer Institute. ESR2 Status by Immunohistochemistry. NCI Thesaurus. Code C157197.

An indication that expression or absence of ESR2 in a sample was determined using immunohistochemical staining techniques. 